\title{
Conocimientos desplegados por estudiantes de pedagogía en Educación Primaria al Diseñar una Tarea Matemática
}

\author{
Knowledge displayed by Pedagogy Students in Primary Education when \\ Designing a Mathematical Task
}

Eugenio Chandia*

ORCID iD 0000-0003-2489-1226

Anahí Huencho **

ORCID iD 0000-0001-6114-5332

Hernán Rivas***

ORCID iD 0000-0002-5847-0756

Andrés Ortíz ${ }^{* * * *}$

ORCID iD 0000-0003-1370-8051

\begin{abstract}
Resumen
Entre las actividades que desarrolla un profesor para enseñar matemática se encuentra diseñar y ajustar Tareas Matemáticas (TM). Para esto, la Formación Inicial Docente (FID) debe proveer de oportunidades y recursos a los futuros profesores para que puedan diseñar TM exitosas. Por lo anterior, esta investigación determinará los conocimientos que 66 estudiantes de pedagogía de Educación Primaria de sexto semestre despliegan al diseñar una TM. A través de la aplicación de Análisis de Clases Latentes se encontraron tres grupos de conocimiento que determinaron tres tipos de TM diferentes: Conceptuales, Procedimentales y Genéricas. Entre los tres grupos, la TM procedimental es la que presenta mayor proporción entre las actividades descritas por los estudiantes, alcanzando un $51 \%$ de la muestra.
\end{abstract}

Palabras clave: Formación inicial docente. Diseño de Tareas Matemáticas. Conocimiento de futuros profesores.

\begin{abstract}
* Profesor de Matemática y Computación, Universidad de Concepción (UDEC). Académico de la Universidad de Concepción, Concepción, Octava Región del Bío-Bió, Chile. Dirección postal: Victor Lamas 1290, Concepción, Concepción, Chile, CP: casilla 160-C. E-mail: echandia@udec.cl.

** Profesor de Estado en Matemática, Universidad de la Frontera (UFRO). Investigadora Doctoral del Centro de Estudios Interculturales e Indígenas (CIIR), Santiago, Región Metropolitana, Chile. Dirección postal: Av. Vicuña Mackenna 4860, Macul, CP: 7820-436. E-mail: aahuencho@uc.cl.

***Profesor de Educación General Básica con Mención en Matemática, Pontificia Universidad Católica de Chile (PUC). Académico de la Pontificia Universidad Católica de Chile - Campus Villarrica. Dirección postal: Novena Región de la Araucanía, Chile. O”Higgins 501, Villarrica, Chile, CP: 4930-000, casilla 12 D. Email: hrivasa@uc.cl.

**** Profesor de Matemática, Universidad de Concepción (UDEC). Académico de la Universidad Católica de la Santísima Concepción (UCSC), Concepción, Región del Biobío, Chile. Dirección postal: Alonso de Ribera 2850, Concepción, Chile, CP: 4090-541. E-mail: aortiz@ucsc.cl.
\end{abstract}


Among the activities that a teacher develops for teaching mathematics is to design and adjust Mathematical Tasks (MT). For this, the Initial Teacher Training (ITT) must provide opportunities and resources to future teachers so they can design successful MTs. Therefore, this research will determine the knowledge that 66 sixthsemester Primary Education pedagogy students deployed when designing a MT. Through the application of Latent Class Analysis, three knowledge groups were found that determined three different TM types: Conceptual, Procedural, and Generic. Among the three groups, the procedural MT is the one with the highest proportion among the activities described by the students, reaching $51 \%$ of the sample.

Keywords: Initial teacher training. Design of Mathematical Tasks. Knowledge of future teachers.

\section{Introducción}

Comúnmente, la enseñanza de las matemáticas está organizada en torno a actividades (DOYLE, 1988), o lo que la literatura actual denomina como Tarea Matemática (TM), las cuales focalizan la atención de los escolares en una idea matemática. (STEIN et al., 1996; SULLIVAN et al., 2012; WATSON; OHTANI, 2015).

El NCTM (2014) indica que un aprendizaje matemático eficaz se logra por medio de la selección e implementación de TM desafiantes. Para adquirir este conocimiento, la formación de profesores, en especial la formación inicial docente (FID), ha de procurar dar oportunidades de aprendizaje a los futuros profesores que les permitan orquestar tareas matemáticas en el contexto escolar para generar un aprendizaje profundo, significativo y de calidad, de manera que aprendan matemáticas tal como se espera que sus alumnos las aprendan (BOYD et al., 2009; BLÖMEKE et al., 2015; CHAPMAN, 2008).

En Chile, los resultados de evaluaciones de egreso a los programas de formación evidencian que la FID otorga pocas oportunidades de aprendizaje, tanto para que desarrollen habilidades matemáticas como competencias profesionales, que le permitan diseñar una TM, ya que no poseen los conocimientos disciplinares ni didácticos para una enseñanza eficaz (ÁVALOS; MATUS, 2010; MINEDUC, 2015), afectando el proceso práctico de hacer matemática y de enseñanza (MINEDUC, 2005; GAETE et al., 2016).

Ahora, si se considera que la actividad práctica de diseñar TM incrementa el conocimiento matemático y las capacidades didáctico-matemáticas, y que la TM es evidencia de estos conocimientos (THANHEISER, 2015; PEPIN, 2015), se puede conjeturar que los futuros profesores no han experimentado el proceso de diseñar una TM, por falta de conocimiento didáctico-matemático o por la carencia de oportunidades para hacerlo.

En consecuencia, este estudio buscó conocer y relacionar los conocimientos que los estudiantes de pedagogía en Educación Primaria evocan y plasman al diseñar TM, caracterizando y tipificando las tareas que resultan de la evocación de uno u otro 
conocimiento como efecto directo de las oportunidades obtenidas en la FID.

Los resultados de este trabajo dan ejemplos de las capacidades de los futuros profesores para diseñar TM, lo que debería considerarse en el proceso de FID, proponiendo nuevas líneas de trabajo en torno a ello. De lo anterior, las preguntas que orientaron la investigación fueron: ¿Qué conocimientos despliegan los estudiantes de Pedagogía de Educación Primaria al diseñar una TM?, ¿Existen grupos de estudiantes de pedagogía con uso de conocimientos comunes? Si los hay, ¿cuáles son estos conocimientos y cómo se relacionan? Y, por último, ¿el tipo de conocimiento desplegado determina algún tipo de TM?

\section{Marco Conceptual}

Para comprender los resultados que se presentan en este artículo, es necesario profundizar en los conocimientos que deben adquirir los futuros profesores de Educación Primaria en el proceso de Formación Inicial Docente (FID).

\subsection{Conocimientos que se deben adquirir en la FID}

La Competencia Profesional Docente (CPD) (BLÖMEKE et al., 2010; KUNTER et al., 2013; WEINERT, 2001) se define como lo que debe lograr el proceso de FID. Esta competencia se compone por capacidades cognitivas y de habilidad para resolver problemas en el aula, tales como conocimiento de la disciplina, conocimiento pedagógico, conocimiento didáctico del contenido, y habilidades de percepción, interpretación y diagnóstico (SHULMAN, 1986; BLÖMEKE et al., 2016; WEINERT, 2001).

En particular, entre las capacidades cognitivas Tatto et al. (2008) plantean en el proyecto Teacher Education and Development Study: Learning to Teach Mathematics (TEDSM) una serie de aristas de la CPD: Conocimiento del Contenido Matemático, que incluye el conocimiento profundo de la matemática escolar; Conocimiento Pedagógico del Contenido matemático, que aborda el conocimiento sobre planificaciones y diseños de clases, conocimiento interactivo aplicado a situaciones de enseñanza, como también el conocimiento del currículo escolar, tal como lo plantea Shulman (1986).

En cuanto al conocimiento sobre planificaciones y diseños de clases, Tatto et al. (2012) afirman que es necesario observar, en los programas de formación de profesores, que los estudiantes de pedagogía puedan: seleccionar apropiadamente actividades; predecir 
respuestas típicas de los niños y niñas, lo que incluye errores y dificultades; planificar métodos apropiados para representar ideas matemáticas; relacionar métodos didácticos con diseños instruccionales; identificar diferentes aproximaciones a la resolución de problemas; seleccionar métodos e ítems para evaluar.

En cuanto al conocimiento curricular, se establece que los estudiantes de pedagogía deben conocer el currículo escolar de matemática; establecer objetivos adecuados; identificar ideas claves en los programas de aprendizaje; seleccionar patrones posibles y visualizar conexiones en el currículum; conocer diferentes formatos de evaluación.

Por último, en cuanto al conocimiento para promover la matemática y su aprendizaje, los autores esperan que los estudiantes de pedagogía puedan representar o explicar conceptos o procedimientos matemáticos; generar preguntas productivas; diagnosticar respuestas de niños y niñas, incluyendo errores y dificultades; analizar o evaluar soluciones o argumentos; analizar el contenido de las preguntas; responder a ideas inesperadas; proveer de retroalimentaciones apropiadas.

\subsection{Tarea Matemática: definición, tipos y fases de trabajo}

Doyle (1983) definió actividades académicas como aquellas que esperan productos de los escolares relacionados a propósitos escolares $\mathrm{y}$, por tanto, permiten explicar las conexiones entre enseñanza y aprendizaje que se producen en una clase (STEIN et al., 1996). Hiebert y Wearne (1993) afirman que una gran cantidad del aprendizaje que un niño o niña adquiere, está definido por los tipos de actividades que ellos hacen. Así, las actividades de clase, cumplen un rol mediador entre lo que se quiere que aprendan los escolares y cómo esto se puede lograr, desde el diseño de la actividad, hasta lo que el estudiante producirá y aprenderá. De esta forma, Stein et al. (1996) definieron una TM como una o varias actividades de clase que focalizan la atención de los escolares en una idea matemática.

\subsubsection{Tipos de Tareas Matemáticas}

Las tareas pueden ser diversas y clasificables, Stein et al. (1996) plantean que una primera categorización de las tareas responde al tipo de uso que estas tengan. Si la tarea tiene la intención de promover el aprendizaje, entonces tiene un carácter instruccional, y si la tarea tiene la intención de generar información sobre el aprendizaje de los escolares, o sobre la 
efectividad de la instrucción, entonces tiene un carácter evaluativo. Una tarea también puede servir para ambos propósitos (WILLIAMS; CLARKE, 1997).

Otra clasificación que se observa en la literatura responde a criterios que consideran el hacer del sujeto que se enfrenta a la tarea y lo que ésta les demanda (SHIMIZU et al., 2010). Entre estas, destacan tres categorías: tareas auténticas, complejas y ricas. La clasificación de auténtico, según los autores, tiene varias acepciones, entre las cuales destaca la naturaleza de la tarea matemática en relación a otro tipo de actividades matemáticas que se pueden realizar fuera del aula escolar con propósitos lejanos al aprendizaje de la matemática.

Las tareas complejas responden a varias características como lo son el lenguaje, el contexto, ámbito numérico, conceptos, representaciones, operaciones, grado de abertura, demanda cognitiva, características en la tarea, currículo escolar, expectativas sociales, entre otras y de las producciones esperadas de los escolares (WILLIAMS; CLARKE, 1997). Una tarea matemática se clasifica como rica si esta admite una gran variedad de acercamientos, permitiendo revelar diferencias en las concepciones de los escolares respecto de conceptos y procedimientos matemáticos.

Así, una tarea puede variar no solo en el contenido o implementación por parte de los profesores, sino también pueden diferenciarse en función de los procesos cognitivos involucrados. Doyle (1988) plantea que tareas con diferentes demandas cognitivas pueden inducir diferentes tipos de aprendizaje, lo cual dependerá del tipo de oportunidades que estas den a los escolares.

En esta línea, Stein et al. (1996) plantean que las demandas cognitivas de las tareas matemáticas pueden cambiar en función de cómo estas se presentan, o bien en cómo son ejecutadas, describiendo una posible trayectoria del desarrollo de una tarea en el aula escolar. Así, la demanda cognitiva de una tarea matemática depende tanto de las características propias de ésta, de cómo se implementa y de lo que hacen quienes la implementan.

\subsubsection{Fases de trabajo de tareas matemáticas}

Stein et al. (1996) proponen una secuencia que relaciona el aprendizaje de los escolares, el diseño y la implementación de tareas matemáticas con un carácter instruccional. Esta secuencia se evidencia en tres fases: en la primera fase la tarea pasa por el currículo o por los materiales instruccionales; en la segunda por el profesor en la clase; y en la última, por la implementación por parte de los escolares (Figura 1). 


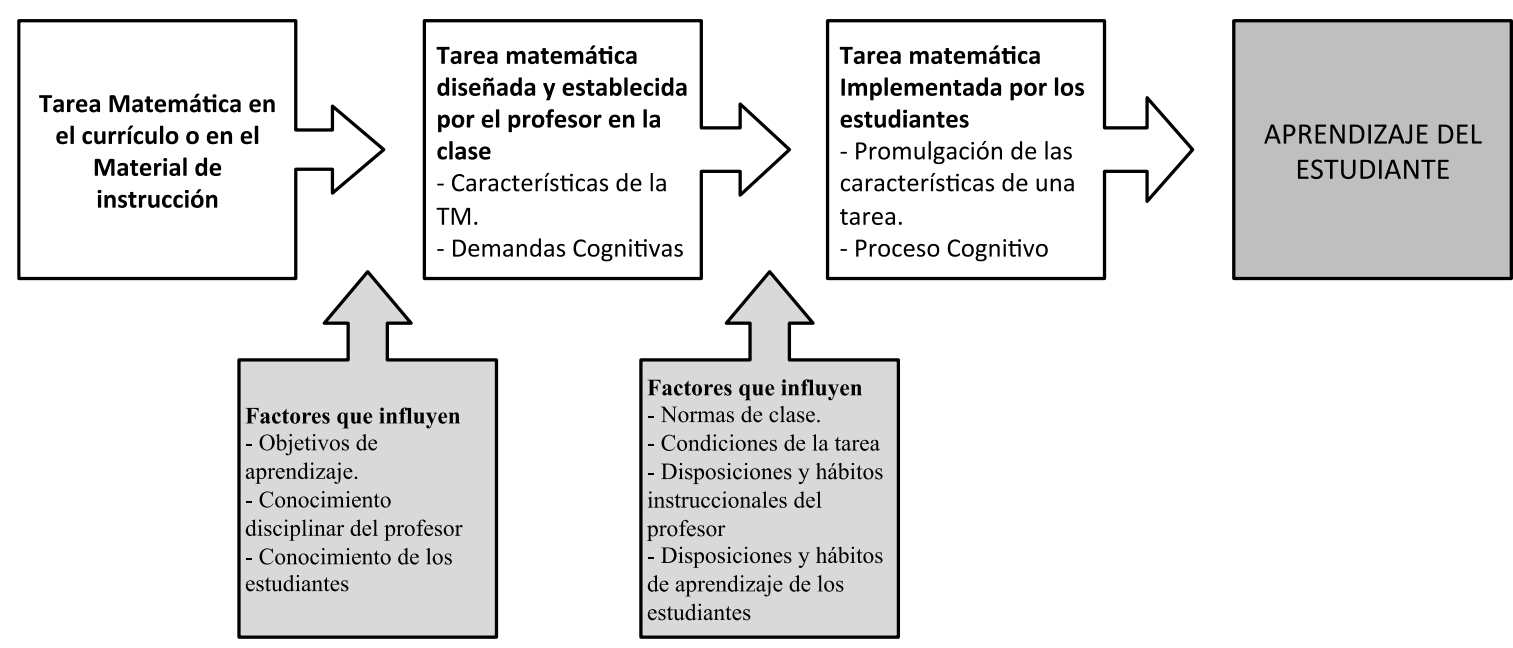

Figura 1 - Secuencia para el desarrollo e implementación de tareas con carácter instruccional Fuente: Stein Grober y Henningsen (1996, p.459)

En la primera fase, la TM se observa en el marco curricular que orienta las ideas sobre las cuales se deberá enfocar la atención de los escolares. Esto deberá orientar e influir la configuración de la tarea, tanto como los materiales e insumos de los que disponga el profesor para implementarla (DOYLE, 1983; STEIN et al., 1996).

En la fase de diseño y establecimiento se configura la tarea, la cual se ve influenciada por los objetivos de enseñanza, el conocimiento del contenido disciplinar del profesor y el conocimiento que tenga el profesor sobre sus alumnos en relación a la TM. En esta fase también se establecen las normas de trabajo, el uso de recursos y materiales, las orientaciones verbales de cómo abordar la tarea y discusiones respecto de lo que se espera de los escolares en relación a la tarea mediante instrucciones cortas (STEIN et al., 1996).

Por otra parte, la demanda cognitiva se refiere al tipo de pensamiento que conlleva resolver la tarea anunciada por el profesor. El rango de tipos de pensamiento va desde la memorización, el uso de procedimientos y algoritmos, con o sin comprensión de conceptos, hasta el empleo de pensamiento complejo y razonamiento estratégico que implica el hacer matemática.

Por último, la fase de implementación es cuando los escolares trabajan sobre la TM. Esta fase se ve influenciada por las normas de clases, la condición de la tarea, los hábitos y disposiciones instruccionales del profesor, más hábitos y disposiciones de aprendizaje de los escolares (STEIN et al., 1996). En esta fase, se ponen en la escena del estudiante las características de la tarea, tales como el lenguaje y el contexto. En paralelo a esto, la demanda cognitiva potencial de la tarea, configurada en la fase anterior, se transforma en demanda cognitiva real, a través de los procesos que los escolares realizan al abordar la tarea. 
Entre los factores que influencian que una tarea baje de nivel al ser implementada, están que estas no sean desafiantes, poco apropiadas a los escolares, clases focalizadas en la respuesta correcta, mucho o poco tiempo dado a la tarea, falta de responsabilidad en el aprendizaje o en la enseñanza, gestión de problemas, entre otros aspectos (STEIN; SMITH, 1998).

Por otra parte, algunos de los factores que influencian el aumento de complejidad de una tarea al ser implementada son: construcción o desarrollo de una tarea considerando el conocimiento de los escolares, tiempo apropiado para el desarrollo de la tarea, modelo de alto desempeño, presión constante sobre los escolares para explicar y dar significado, andamiaje, auto-monitoreo de los escolares, o bien que el profesor muestre las conexiones conceptuales (BOSTON et al., 2015).

\subsection{Efecto del diseño de tareas en la formación de profesores}

El profesor, al diseñar TM e implementarlas, deja evidencia de su conocimiento matemático y percepción sobre el aprendizaje y la enseñanza de la matemática (HAGGARTY; PEPIN, 2002; SCHMIDT et al., 1997), permitiendo, entonces, observar sus dificultades o errores conceptuales. De esta forma, investigadores afirman que los procesos de desarrollo profesional deberían focalizarse en apoyar a los profesores en reconocer la complejidad de tomar decisiones al diseñar TM e implementarlas (CLARKE et al., 2009; ZASLAVSKY; SULLIVAN, 2011).

En la FID, al diseñar TM, el futuro profesor puede acceder al pensamiento matemático de los escolares, al pensar en la posible interacción de la TM con ellos, determinando posibles trayectorias de pensamiento al enfrentarlos a la TM (JAWORSKI, 2007; PEPIN, 2015; ZASLAVSKY; SULLIVAN, 2011); de esta forma, los futuros profesores se adentran al triángulo didáctico que se presenta en la sala de clases.

El diseño TM en la FID puede influir en la estructura de las posibles capacidades de diseño de los futuros profesores (THANHEISER, 2015). Pepin (2015) y Pepin et al. (2013) afirman que, cuando los docentes interactúan con las TM, se pueden observar que ellos: adaptan e integran materiales curriculares; modifican sus prácticas y su conocimiento matemático; cambian sus capacidades didácticas pedagógicas; se ven influenciados por los recursos curriculares en cuanto a posibles secuencias de TM. Por otra parte, los autores también observan que la calidad de las TM se ven afectadas por las capacidades y 
conocimientos de los docentes, por lo que el efecto es bidireccional, el profesor se ve afectado por la TM y la TM por el profesor.

\section{4 ¿Qué necesita el profesor para diseñar tareas?}

Watson y Ohtani (2015) declaran que al diseñar TM se debe considerar: a) ¿qué se diseña? (tareas individuales; grupales; secuencia de tareas), b) ¿cuáles son las herramientas necesarias?, recursos manipulables, tecnológicos, recursos cognitivos, c) bajo qué condiciones se puede diseñar una TM. El diseño se hace de forma individual, de forma colectiva con otros colegas, quienes están involucrados en la TM, cuáles es el propósito de la tarea etc.

Por otra parte, Liljedahl et al. (2007) considera que el profesor debiera seguir las siguientes etapas para diseñar TM: anticipar; diseñar; implementar; reflexionar; modificar y rediseñar. En el análisis predictivo el profesor o futuro profesor debiera recurrir a su experiencia personal, como también a experiencias con tareas similares. Al diseñar, el profesor considera su análisis predictivo y lo bosqueja en un plan para ser implementado. Esto puede usar diferentes tipos de conocimiento matemático como didáctico.

En la implementación, la TM se ve enfrentada a un proceso de interacción entre la TM, el profesor y los escolares, lo cual puede generar modificaciones in situ o posteriores a la TM. En el análisis reflexivo, se evalúan las decisiones pedagógicas acordadas en función de los resultados de la implementación. Por último, se realizan ajustes donde, nuevamente, se reflexiona y evalúa todo el proceso.

De esta forma, el diseño TM no requiere solamente de habilidades o capacidades pedagógicas y conocimientos matemáticos, requiere del conocimiento de recursos curriculares y de la capacidad para percibir e interpretar la interacción entre la TM y los niños y niñas con el propósito de tomar decisiones que enriquecerán el diseño TM.

\section{Metodología}

Dado que esta investigación tiene como propósito explorar y relacionar conocimientos que ponen en juego estudiantes de pedagogía al diseñar una TM, es que se adopta un diseño mixto (cuali-cuanti) de carácter descriptivo y relacional. Primero, se realizó un Análisis del Contenido por medio de un proceso de codificación de las actividades en formato escrito, descritas por los estudiantes de pedagogía al diseñar una TM, considerando categorías de 
conocimiento que resultaron de la revisión de literatura, descrito en el marco de antecedentes, potenciadas con categorías que emergen del proceso. Luego se realizó un análisis descriptivo de las categorías observadas para, finalmente, aplicar un Análisis de Clases Latentes con el cual se determinaron relaciones entre las categorías de conocimiento de los estudiantes de pedagogía al diseñar una TM.

En esta investigación participaron 105 estudiantes de pedagogía de Educación Primaria, que respondieron un cuestionario sobre prácticas de instrucción en Matemática de tres instituciones universitarias de Chile, con 5, 6 y 7 años de acreditación institucional. Del total, solo 66 describieron la actividad y corresponden al total de casos analizados de esta investigación. Al momento de aplicar el instrumento los estudiantes de pedagogía habían cursado entre cinco y seis cursos de Matemática o Didáctica de las Matemáticas de un total de entre siete y diez cursos según sus respectivas mallas curriculares, focalizados en contenidos escolares como los conjuntos de los números Naturales y Enteros, en conocimientos de Geometría Euclidiana, Identificación de Patrones, Generalizaciones y Lenguaje Algebraico, su enseñanza y aprendizaje.

Con el propósito de profundizar en los conocimientos que evocan los estudiantes de pedagogía al diseñar una TM se utilizaron viñetas, que consisten en poner a los entrevistados en una situación ficticia (SCHOENBERG; RAVDAL, 2000) y a partir de ella realizar preguntas del tema que se quiere conocer tal como se describe en el Cuadro 1. Tal viñeta se les presentó a los estudiantes de pedagogía en cuestionario en una clase regular del proceso FID, con el permiso del académico formador, donde tuvieron 45 minutos para completar todo lo que les solicitaba.

\begin{tabular}{|l|}
\hline Situación descrita a los estudiantes de pedagogía \\
\hline La profesora Valeria hace clases de matemática a un tercero básico. Ella debe abordar el siguiente objetivo de \\
aprendizaje con sus estudiantes: "Demostrar que comprenden la adición y la sustracción de números del 0 al \\
1 000. creando y resolviendo problemas de adición y sustracción". La profesora quiere dar la oportunidad al \\
estudiante en práctica para que implemente solo una actividad matemática con sus alumnos que no dure más \\
de 30 minutos. (...) ella le pide que diseñe una actividad para abordar el objetivo de aprendizaje o parte de él \\
en 30 minutos de una clase. (...) que presente en no más de página y media lo que va hacer, considerando la \\
tarea matemática que va trabajar con los niños, las posibles orientaciones para generar interacciones y \\
discusiones y retroalimentaciones o devoluciones que entregará a los diferentes tipos de producciones de los \\
estudiantes. ¿Qué actividad matemática propondrías? Describe en el siguiente espacio lo que le propondrías a \\
la profesora Valeria hacer con sus estudiantes.
\end{tabular}

Cuadro 1 - Viñeta utilizada en el cuestionario Fuente: Elaborado por los autores

\subsection{Análisis de los datos}

Para analizar cada una de las actividades de forma escrita, se procedió a realizar un 
análisis centrado en lo que el texto dice en función al papel desempeñado por el conocimiento teórico existente, con diferentes grados de rigidez. Así, por medio de un Análisis del Contenido Cualitativo (MAYRING, 2014), se realizó una primera codificación por dos correctores especializados como proceso de calibración y fortalecimiento de las categorías obtenidas de la revisión de literatura, específicamente de los apartados 2.1, 2.2 y 2.3 del marco conceptual, permitió incorporar elementos emergentes a ésta.

Las categorías y los descriptores que se establecieron para el análisis se observan en el Cuadro 2. Luego se procedió a realizar una doble corrección formativa y, por último, la doble corrección definitiva. Todas las dudas y discrepancias del proceso fueron revisadas por un investigador experto. El índice Kappa se calculó con el 100\% de los datos. Una vez alcanzada la consistencia se procedió a corregir individualmente. Los índices Kappa variaban entre 0.91 y 1.0 con un promedio de 0.96 .

\begin{tabular}{|c|c|}
\hline Categorías & Indicadores \\
\hline $\begin{array}{l}\text { Conocimiento } \\
\text { Matemático } \\
\text { (CM) }\end{array}$ & $\begin{array}{l}\text { Conceptual (CMC): aquel que profundiza en el conocimiento matemático, realiza } \\
\text { conexiones ricas entre conceptos, definiciones, teoremas o algoritmos, permitiendo una } \\
\text { comprensión flexible del conocimiento (HIEBERT; LEFEVRE, 1986). Procedimental } \\
\text { (CMP): aquel que se preocupa de secuencias de acciones o pasos para realizar una } \\
\text { actividad matemática. }\end{array}$ \\
\hline $\begin{array}{l}\text { Conocimiento } \\
\text { del Estudiante } \\
\text { (CE) }\end{array}$ & $\begin{array}{l}\text { Errores (CEE): considera errores matemáticos que pueden cometer los estudiantes. } \\
\text { Dificultades u Obstáculos (CEDO): elementos que impiden que el estudiante avance en } \\
\text { estrategias o soluciones de origen matemático o bien metodológico. Conocimiento Previo } \\
\text { (CECP): considera aprendizajes adquiridos con anterioridad en el estudiante. Habilidades } \\
\text { (CEH): considera las habilidades de los alumnos para abordar actividades o situaciones } \\
\text { matemáticas. Capital Cultural (CECC): considera el contexto cotidiano potencial en las } \\
\text { actividades. Aspectos Afectivos (CEA): provocan el interés, las actitudes y emociones } \\
\text { hacia el aprendizaje del contenido matemático de los estudiantes. }\end{array}$ \\
\hline $\begin{array}{l}\text { Conocimiento } \\
\text { del currículo } \\
\text { escolar }(\mathrm{CCE})\end{array}$ & $\begin{array}{l}\text { Descompone el Objetivo de Aprendizaje (CCEDOA): el objetivo dado se descompone en } \\
\text { variadas habilidades y conocimientos matemáticos que aportan al logro del OA. } \\
\text { Conexiones dentro del Nivel (CCECX): se hacen conexiones entre contenidos } \\
\text { matemáticos y de otras áreas como ciencias desarrollados en el mismo nivel de enseñanza. } \\
\text { Conexiones entre diferentes Niveles (CCEONIV): Se hacen conexiones entre los } \\
\text { conocimientos de otros niveles y el conocimiento del objetivo de aprendizaje. } \\
\text { Incorporación de Metas (CCEILOGRO): se establecen metas, objetivos o sub-objetivos de } \\
\text { aprendizaje que permiten cumplir el objetivo general. }\end{array}$ \\
\hline $\begin{array}{l}\text { Conocimiento } \\
\text { para gestionar } \\
\text { una clase } \\
\text { (CGC) }\end{array}$ & $\begin{array}{l}\text { Prácticas de Interacción (CGCPI): interacción entre el profesor y los estudiantes, como la } \\
\text { retroalimentación a las contribuciones de los estudiantes, o bien, entre los propios } \\
\text { estudiantes. Prácticas de Discusión (CGCPD): centrada en la discusión de la veracidad o } \\
\text { falsedad de afirmaciones. Razonamiento Matemático (CGCRM): prácticas para mantener } \\
\text { y provocar el RM del estudiante. Tiempo (CGCTIEM): consideración del tiempo en el } \\
\text { desarrollo potencial de las actividades. Organización de la Clase (CGCOCLASE): } \\
\text { consideración de la organización de los alumnos en la sala de clases durante } \\
\text { implementación de la TM. }\end{array}$ \\
\hline Recursos (R) & $\begin{array}{l}\text { En la descripción se observa la consideración de material concreto (RMC), material } \\
\text { tecnológico (RMTIC), guías o textos escolares (RGT). }\end{array}$ \\
\hline $\begin{array}{l}\text { Tipo de } \\
\text { problema (TP) }\end{array}$ & $\begin{array}{l}\text { Rutinario (TPR): en el sentido que propongan estructuras procedimentales, de prácticas o } \\
\text { de ejercitación común. No Rutinarios (TPNR): que provoquen a los alumnos a razonar } \\
\text { matemáticamente. Abiertas o ricas (TPA): en relación a las posibilidades de abordar } \\
\text { diferentes estrategias o soluciones posibles. Semi-abierta o semi-ricas (TPSA): en donde } \\
\text { la tarea presenta limitaciones de estrategias o soluciones para abordar o solucionar la } \\
\text { actividad. }\end{array}$ \\
\hline
\end{tabular}




\begin{tabular}{|l|l|}
\hline Nivel de & Memorísticas (NCPM): que solo se focalizan en la reproducción de contenidos, reglas o \\
Complejidad & definiciones, de manera directa. Procedimientos sin conexión (NCPPSC): donde se usan \\
Potencial de la & procedimientos aprendidos o bien dados en la misma actividad. Procedimientos con \\
TM (NCP) & $\begin{array}{l}\text { conexión (NCPPCC): donde se profundiza en la comprensión de los conceptos o ideas } \\
\text { clave presentes en los procedimientos. El hacer matemático (NCPHM): donde se deba } \\
\text { usar un pensamiento complejo y no algorítmico, se promueva la exploración, comprensión } \\
\text { de conceptos y la autorregulación, evaluando las limitaciones o alcances de las soluciones } \\
\\
\text { o estrategias para abordar la actividad. }\end{array}$ \\
\hline
\end{tabular}

Cuadro 2 - Categorías de clasificación de la demanda potencial de una tarea

Fuente: elaborado por los autores

Una vez codificados la totalidad de los casos, se procede a analizar la codificación, realizada de manera descriptiva, a nivel de categoría y de sus indicadores. Luego, se analizan los casos según características comunes usando el Análisis de Clases Latentes (ACL) para identificar asociaciones entre sujetos (casos) que comparten características o conductas similares (ROST, 2004; MAGIDSON; VERMUNT, 2004), como descripciones de TM.

Comparado con los análisis clásicos de análisis de clúster, el ACL realiza clasificaciones basado en probabilidades (los casos o sujetos son clasificados en un clúster basados en la probabilidad estimada de pertenecer a éste). Sujetos o casos agrupados de acuerdo a los resultados del ACL son denotadas como clases latentes. Conceptos educacionales que no pueden ser medidos directamente como conductas, creencias o estilos de prácticas de instrucción pueden ser modelados y caracterizados usando indicadores variables (KÖNIG; BLÖMEKE, 2012).

De esta forma, cada uno de los indicadores de conocimiento necesarios para diseñar una TM, que fueron obtenidos de la revisión literatura y de elementos emergentes del Análisis del Contenido Cualitativo, pueden ser agrupados en función de la distribución de presencia o ausencia en las descripciones de las TM de los estudiantes de pedagogía, generando relaciones entre ellos y, por ende, entre las clases latentes que los diferencian y los agrupan en función del diseño la TM. El análisis fue hecho con la función poLCA del paquete del mismo nombre de programa R-project (FINCH; FRENCH, 2015).

La decisión sobre el número de clases fue hecha en base al criterio de información Akaike y el criterio de información Bayesiana (AIC y BIC por sus siglas en inglés respectivamente), considerando que el menor valor en ambos criterios indica un modelo con mejor ajuste (ROST, 2004).

\section{Resultados}

\subsection{Análisis descriptivo}


En la Figura 2 se observa que las categorías de conocimiento menos observadas en las actividades fueron: conocimiento del estudiante, principalmente aquellas relativas al conocimiento de errores y dificultades u obstáculos; conocimiento del currículo escolar relativo a la consideración de conocimientos de otras áreas disciplinares, como también de otros niveles escolares del eje de matemática; mención al uso de recursos tecnológicos; conocimiento de tipos de problemas abiertos y caracterizar la TM para que los escolares hagan matemática en términos de la complejidad cognitiva.

Por otra parte, las categorías de conocimiento más observadas fueron: TM con foco en lo procedimental; conocimientos de prácticas de interacción; conocimiento sobre la organización de clase; uso de materiales concretos como recursos; uso de actividades rutinarias y no rutinarias y TM del nivel de complejidad procedimental sin conexiones.

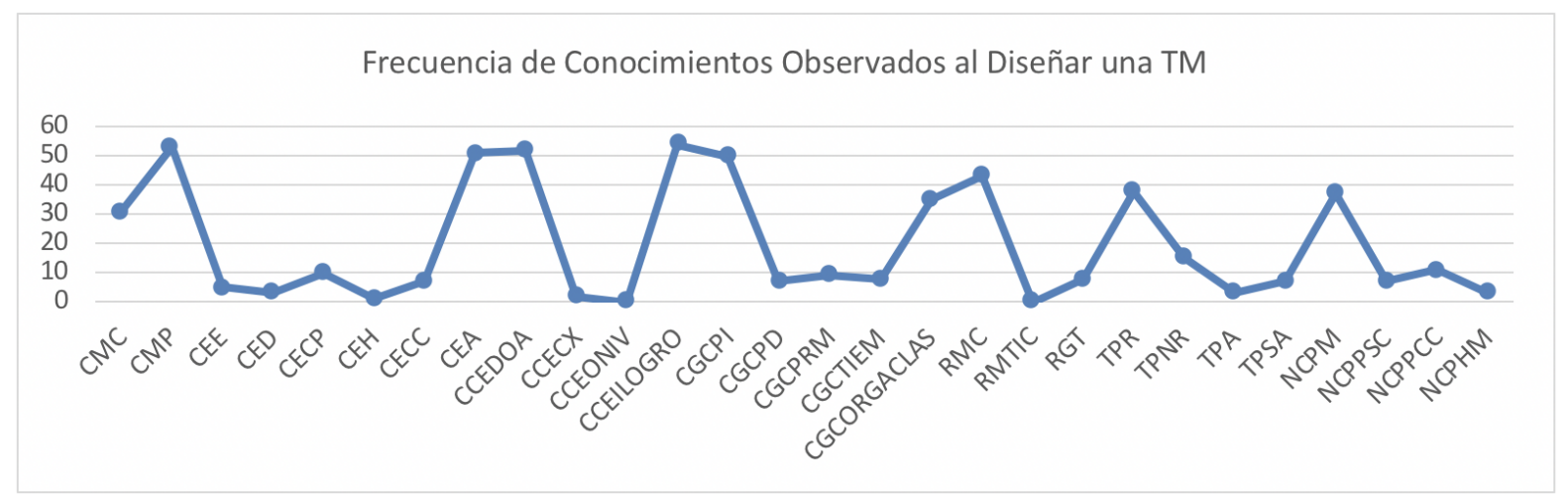

Figura 2 - Frecuencia absoluta de conocimientos observados en las TM diseñadas por los estudiantes de Pedagogía en Educación Básica Fuente: elaborado por los autores

Al observar la frecuencia relativa entre la presencia de los indicadores de cada una de las dimensiones se puede observar que, en el 50\% de las tareas diseñadas por los estudiantes de pedagogía, es posible identificar al menos un tipo de conocimiento matemático. Por otra parte, se observa que las dimensiones de conocimiento relativo a los estudiantes, al currículo escolar, la gestión de clases y recursos tienen menos de un $40 \%$ de presencia en las tareas diseñadas donde la frecuencia es de a lo más 2 indicadores. En cuanto al tipo de actividad y potencial de complejidad de las tareas, se observa que ambas tienen la misma distribución de observación en las tareas, con presencia de a lo más dos indicadores en cada una de las dimensiones. 


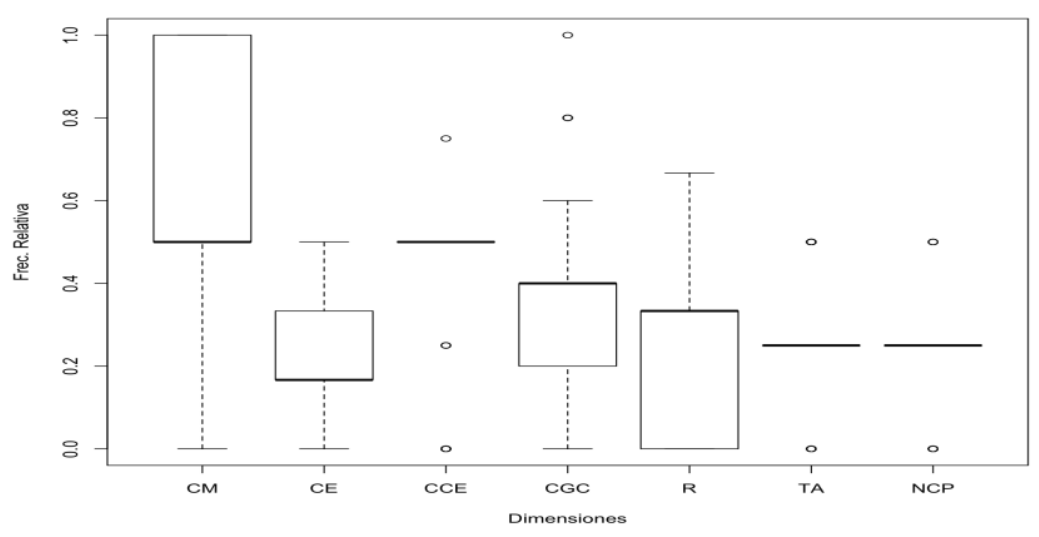

Figura 3 - Distribución de las frecuencias relativas entre el número de indicadores de conocimiento observados y el esperado en cada dimensión por actividad diseñada

Fuente: elaborado por los autores

\subsection{Análisis de Clases Latentes de todas las categorías}

Se estimaron tres modelos con dos, tres y cuatro clases latentes posibles, de los cuales el modelo con tres obtuvo el menor valor para ambos criterios de información, tal como se observa en la Tabla 1.

Tabla 1 - Criterios de información AIC y BIC

\begin{tabular}{ccc}
\hline Modelo & AIC & BIC \\
\hline Dos clases latentes & 1364.45 & 1479.69 \\
Tres clases latentes & 1231.17 & 1405.12 \\
Cuatro clases latentes & 1272.55 & 1505.21 \\
\hline
\end{tabular}

Fuente: elaborado por los autores

La Figura 4 muestra la distribución de probabilidad de cada una de las categorías de conocimiento de pertenecer a una u otra clase.

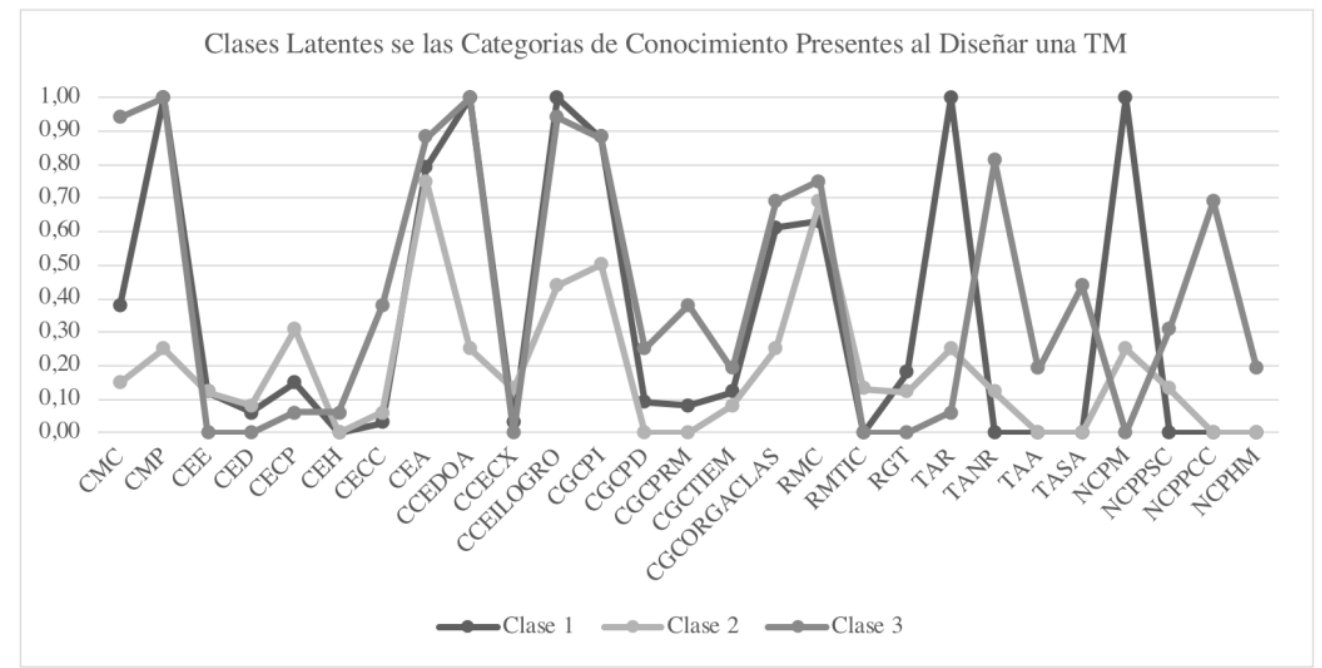

Figura 4 - Clases Latentes de Estudiantes de Pedagogía de Educación Básica al Diseñar una TM Fuente: elaborado por los autores 
La probabilidad que un estudiante pertenezca a la Clase 1 es de un 51\%; de pertenecer a la Clase 2 y Clase 3, del 25\% respectivamente.

Clase 1 - Procedimentales. Esta clase latente se caracteriza por estudiantes de pedagogía que, al diseñar una TM para el objetivo de aprendizaje dado, se focalizan, principalmente, en abordar conocimientos matemáticos procedimentales $(\mathrm{P}(\mathrm{CMP} / \mathrm{C} 1)=1$ probabilidad de presentar la categoría CMP, condicionado a estar en la clase 1-), por ejemplo, se pueden observar actividades del estilo:

Hacer una suma y resta, enseñar cómo hacer una suma, responder dudas, enseñar restas, responder dudas, repaso.

(Estudiante 32, 2016).

En sus actividades se puede observar que son capaces de descomponer el objetivo de aprendizaje dado $(\mathrm{P}(\mathrm{CCEDOA} / \mathrm{C} 1)=1)$ en indicadores de logro específicos los cuales se transforman en sus propósitos de actividad (P(CCEILOGRO/C1) = 1):

Inicio: conocer ideas previas de los niños mostrar un video sobre adiciones, comentar con los niños la importancia de las adiciones en la vida diaria que los niños den ejemplos de su uso en la vida cotidiana. Desarrollo: Plantear situaciones problemas donde se trabaje la adición con material concreto (en parejas). Luego comentar como lo hicieron. Cierre: comentar lo aprendido.

(Estudiante 62, 2016).

Tal como se observa, los estudiantes de pedagogía consideran la organización de los niños y niñas en el desarrollo de la actividad (P(CGCORGACLAS/C1) = 0.61) y el uso de material concreto $(\mathrm{P}(\mathrm{RMC} / \mathrm{C} 1)=0.63)$. Si bien consideran prácticas para la interacción entre el profesor y sus alumnos o entre alumnos $(\mathrm{P}(\mathrm{CGCPI} / \mathrm{C} 1)=0.61)$, o consideraciones afectivas para motivar a los alumnos $(\mathrm{P}(\mathrm{CEA} / \mathrm{C} 1)=0.73)$, sus actividades son rutinarias $(\mathrm{P}(\mathrm{TAR} / \mathrm{C} 1)=$ 1) donde solo se les solicita a los niños y niñas evocar conocimiento, sin realizar conexiones o profundizar en las ideas o conceptos matemáticos $(\mathrm{P}(\mathrm{NCPM} / \mathrm{C} 1)=1)$ :

Desarrollo 6 bloques de 5 minutos con las siguientes indicaciones. Primer bloque juntarse en grupos. Segundo bloque pasar materiales e indicaciones de la actividad (desarrollar una suma y una resta en una cartulina y papeles lustres). Tercer bloque ellos eligen los números con los que realizaran la actividad. Cuarto y quinto bloque exposición al curso de su creación en conjunto y explicar el procedimiento de suma y resta en cada uno. Sexto bloque felicitar a los alumnos y ver qué tan contentos quedaron con la actividad.

(Estudiante 35, 2016).

Clase 3 - Conceptuales. Esta clase se caracteriza por estudiantes de pedagogía que, si bien comparten algunas características con la Clase 1, como que las actividades abordan aspectos procedimentales $(\mathrm{P}(\mathrm{CMP} / \mathrm{C} 3)=1)$, estos muestran actividades donde la intensión es la comprensión conceptual de los conocimientos matemáticos $(\mathrm{P}(\mathrm{CMC} / \mathrm{C} 3)=0.94)$ que se desprenden del objetivo de aprendizaje dado. Las actividades son no rutinarias (P(TANR/C3) 
$=0.81)$, con alta probabilidad de observar instrucciones para establecer conexiones $(\mathrm{P}(\mathrm{NCPPCC} / \mathrm{C} 3)=0.69)$ o ahondar en el conocimiento matemático para mantener a los alumnos razonando matemática $(\mathrm{P}(\mathrm{CGCPRM} / \mathrm{C} 3)=0.38)$ :

La actividad que propondría la utilizaría en el cierre de la clase y trabajaría como refuerzo aplicado al contexto cotidiano de los estudiantes. Esto es sistema monetario. A cada estudiante le entregaría 500 pesos ficticios como sistema del juego monopoly en la pizarra o en la mesa colocaría diferentes objetos que costarán hasta 500. Esos 500 pesos a cada estudiante los entregaría 4 monedas de 100 y 2 de 50 y los precios de las cosas estuvieran a 100,150, 250,350 etc. Les preguntaría ¿Qué objetos puedo comprar con 500 pesos? ¿Cuántos objetos podría comprar? ¿cuánto dinero me queda luego de haber comprado mi primer objeto? ¿Cuántas monedas me quedan? ¿cuánto dinero me queda? Etc. Así, se podría ir sistematizando a través de sus ejemplos las operaciones llevadas a cabo de manera simbólica en la pizarra. Ejemplo: con los 500 pesos me compré un dulce de 250 pesos.

(Estudiante 7, 2016).

Por otra parte, estos estudiantes de pedagogía también comparten con los de la Clase 1 el uso de material concreto $(\mathrm{P}(\mathrm{RMC} / \mathrm{C} 3)=0.75)$, la organización de los niños y niñas en el desarrollo de la actividad $(\mathrm{P}($ CGCORGACLAS/C3) $=0.69)$, prácticas para provocar la interacción entre el profesor y sus alumnos y entre alumnos, como la retroalimentación y preguntas orientadoras en la actividad $(\mathrm{P}(\mathrm{NCPPCC} / \mathrm{C} 3)=0.88)$, y la determinación de subobjetivos o propósitos que se pueden establecer desde el objetivo (P(CCEILOGRO/C3) = 0.94) y se distribuyen y secuencias en la clase para lograr un gran objetivo, que puede ser el dado o bien parte de éste:

Como estudiante en práctica propondría que los alumnos trabajen en parejas creando al menos dos problemas matemáticos (uno de adición y otro de sustracción), para luego intercambiar problemas, resolverlos y discutir bajo las siguientes preguntas. 1-¿Qué operación se asocia al problema? 2-¿Cómo lo supiste? 3-¿Qué elementos del problema te llevaron a establecer esa conclusión? Posteriormente el creador del problema debe retroalimentar a su compañero a partir de lo que el aplicó para crear cada problema. La labor de la profesora recaerá en monitorear el proceso de los alumnos, guiar una las discusiones de cada pareja, dirigir una discusión productiva grupal en donde se discutirán los hallazgos de cada pareja.

(Estudiante 4, 2016).

Sin embargo, estos estudiantes de pedagogía tienen probabilidades mayores o iguales a 0.4 de considerar en sus propuestas de actividades el capital cultural de los niños o niñas $(\mathrm{P}(\mathrm{CECC} / \mathrm{C} 3)=0.4)$, como el contexto o situaciones cotidianas de ellos, lo que relacionan con aspectos afectivos $(\mathrm{P}(\mathrm{CEA} / \mathrm{C} 3)=0.88)$ que tienen como propósito enganchar a los niños y niñas en las TM de las actividades:

Primero se recogerían los conocimientos previos de los alumnos, aclarando que tan interiorizados se encuentran de los números del 0 al 1000 y cubriendo esos contenidos en caso que no exista claridad. Además corroborar la capacidad aditiva y sustractiva de los niños con números pequeños. Posteriormente a ello se establecerían problemas vinculados a su cotidianidad como comprar en el kiosco con $\$ 1000$, el valor de un producto y el vuelto que 
corresponde o las monedas que obtiene en distintas etapas de un video juego, que si las sumara alcanzaría un número menor a 1000. Finalmente, los alumnos exponen sus resultados, se comparan los métodos para llegar a ellos y se establecen cuáles fueron los errores que deben ser corregidos. Primero en pequeños grupos de trabajo y a continuación como curso con la profesora corroborando que la retroalimentación sea correcta.

(Estudiante 70, 2016).

Clase 2 - Genéricos. Los estudiantes de pedagogía que pertenecen a esta clase se caracterizan, principalmente, porque sus actividades no ahondan en el conocimiento matemático conceptual $(\mathrm{P}(\mathrm{CMC} / \mathrm{C} 2)=0.15)$ o procedimental $(\mathrm{P}(\mathrm{CMP} / \mathrm{C} 2)=0.25)$, sin embargo, en sus descripciones se puede observar claramente el uso de material concreto $(\mathrm{P}(\mathrm{RMC} / \mathrm{C} 2)=0.69)$ y de prácticas para provocar la interacción $(\mathrm{P}(\mathrm{CGCPI} / \mathrm{C} 2)=0.5)$ :

Inicio: Estimular a los niños con algo que para ellos sea entretenido, enganchar con la clase anterior. Desarrollo: Mediante un juego plantear un problema desafiante para los niños el cual deban resolver para continuar el juego. Por ejemplo, el docente debe monitorear permanentemente e ir corrigiendo los posibles errores en el acto. Cierre: Preguntar a los niños que fue lo que les resultó más difícil y enseñar diversas estrategias para resolver los posibles problemas. Enganchar con la clase siguiente y mostrar algunos ejemplos de la vida cotidiana donde usarían lo aprendido.

(Estudiante 73, 2016).

Otra característica de este grupo, es que consideran los conocimientos previos $(\mathrm{P}(\mathrm{CECP} / \mathrm{C} 2)=0.31)$ de los alumnos en el desarrollo y descripción de sus clases lo que se transforma en el establecimiento de propósitos o sub-objetivos en sus actividades $(\mathrm{P}(\mathrm{CCEILOGRO} / \mathrm{C} 2)=0.31)$ :

En primera instancia activar los conocimientos previos de los niños sobre los contenidos luego realizar alguna actividad didáctica donde ellos puedan manipular y trabajar con algún implemento o juegos, para luego trabajar resolviendo una guía de ejercicios, realizar constantes retroalimentaciones, pero la más importante al final de la actividad para saber si se logró el objetivo.

(Estudiante 43, 2016).

\section{Discusión y conclusiones}

\subsection{Conocimientos al diseñar una TM}

Independiente de la clase a la cual pueda o no pertenecer un estudiante de pedagogía, los conocimientos relativos a: incentivar, motivar y enganchar a los alumnos con las TM, gestionar clases para provocar la interacción entre alumnos y entre el profesor y sus alumnos; usar recursos como materiales concretos para el trabajo en la TM, permiten establecer que todos los estudiantes de pedagogía al diseñar una TM abordan, principalmente, la transición entre la Fase 2 y Fase 3 para el diseño de una TM que propusieron Stein et al. (1996). 
En este sentido, el proceso anticipatorio (LILJEDAHL et al., 2007) se centra, principalmente, en la dimensión conocimiento del estudiante de pedagogía en cuanto a la atención de los niños y niñas para el desarrollo e implementación de la TM. Si bien esta característica es parte de las que tienen un alto potencial de complejidad cognitiva en el aula escolar de matemática (BOSTON et al., 2015), la ausencia de profundización en el conocimiento matemático del $76 \%$ de las actividades descritas por los estudiantes de pedagogía, da cuenta que los conocimientos desplegados en las actividades no permiten asegurar un potencial cognitivo alto cuando las TM se implementen.

Esto se ratifica por las bajas probabilidades de observar conocimientos relativos a: errores o dificultades posibles de los alumnos en relación al contenido o actividad planteada; las habilidades de los alumnos en relación al posible desempeño de ellos en las actividades; conexiones con otras áreas del conocimiento curricular escolar; prácticas para provocar discusiones matemáticas; el planteamiento de actividades de carácter abierto, conocimientos establecidos para que las TM tengan un nivel alto de complejidad cognitiva en el aula escolar de matemática (BOSTON et al., 2015).

Al observar, de manera diferenciada, los clústeres, se observan conocimientos que se relacionan entre sí, formando una red para diseñar una TM (KÖNIG; BLÖMEKE, 2012). Al observar el clúster 1, se tiene que los conocimientos que surgen en forma conjunta y con altas probabilidades son las relativas a: el uso de conocimientos matemáticos procedimentales; descomposición de objetivos de aprendizaje; establecimiento de indicadores de logro; uso de prácticas de instrucción para generar interacción; uso de actividades rutinarias que se centran en aspectos memorísticos. Ahora, si se considera el marco de análisis de la investigación, los conocimientos suplementarios a los anteriores, que tienen bajas probabilidades de ser observados en esta red son las relativas a: el estudiante en cuanto a errores y dificultades posibles, conocimientos previos, consideración de las habilidades de desempeño de los estudiantes, del capital cultural de éstos, de conexiones con otras áreas de conocimiento, uso de prácticas para provocar discusión y razonamiento matemático y el planteamiento de actividades rutinarias con bajo nivel de conexiones entre los conceptos. Esta red establece un dominio para diseñar cierto tipo de TM que se característica por abordar procedimientos de forma mecánica (WILLIAMS; CLARKE, 1997).

Por otra parte, el clúster 3 determina otra red de conocimientos que se relacionan y que determinan una TM con cierta característica. En particular, los conocimientos que surgen de manera conjunta son: el matemático conceptual y procedimental, del estudiante en cuanto a su 
capital cultural, del currículo escolar respecto de a la descomposición del objetivo de aprendizaje y determinación de secuencias de aprendizaje, de uso de prácticas para gestionar la clase con foco en la promoción del razonamiento matemático, de uso de recursos concretos, el planteamiento de actividades no rutinarias, que permiten hacer conexiones y establecer múltiples soluciones o modos de acercamiento a estos. Estos conocimientos establecen la red para obtener TM ricas, auténticas y complejas (WILLIAMS; CLARKE, 1997; BOSTON et al., 2015). Los conocimientos que tienen bajas probabilidades de salir en esta red los relativos a: el estudiante en cuanto a sus errores, dificultades, conocimientos previos y de habilidades de desempeño, de posibles conexiones con otros conocimientos disciplinares.

Por último, en el clúster 2 se presenta una red de conocimientos relativos al uso de recursos para motivar e incentivar a los alumnos con la TM. Los conocimientos que tienen bajas probabilidades de ser observados son prácticamente todos los definidos en el marco de referencia. Llama la atención la ausencia de conocimiento matemático, como polo opuesta a esta red. De esta forma, esta red es dominio de una nueva categoría de TM, la cual no se observa en la literatura, dado que todas consideran la presencia del conocimiento matemático en la TM (BOSTON et al., 2015; WILLIAMS; CLARKE, 1997).

\subsection{Implicancias para la FID}

Las TM que se puedan diseñar e implementar son clave, dado que son ellas las que direccionan el aprendizaje de los estudiantes, determinando lo que pueden o no aprender en el aula escolar en relación al conocimiento matemático (STEIN et al., 1996). De lo anterior, y dado que un $51 \%$ de los estudiantes de pedagogía de la muestra presentaron actividades de tipo procedimental, los niños y niñas que puedan abordar estas actividades no tendrán oportunidades para adquirir un conocimiento matemático eficaz y profundo (MA, 1999; NCTM, 2014).

Sin embargo, existe un $25 \%$ de los estudiantes de pedagogía que describen TM con potencial de complejidad alto, por lo que habrá niños y niñas que tengan la oportunidad de razonar matemáticamente, interactuar con sus pares en torno actividades abiertas para buscar múltiples estrategias o soluciones a problemas matemáticos. Esto confirma las percepciones de los estudiantes de pedagogía en un hecho concreto como el diseño TM en cuanto a la carencia de oportunidades de tipo práctico, tanto en la disciplina como en la enseñanza para esto (MINEDUC, 2005; GAETE et al., 2016). 
Las TM descritas confirman una carencia de conocimiento metodológico del contenido matemático escolar (ÁVALOS; MATUS, 2010), tanto así que en un $25 \%$ de las actividades descritas no es posible observar ni conocimiento conceptual ni procedimental matemático desplegado en las propuestas de actividad. De esta forma, la habilidad para resolver problemas de aula de estos estudiantes de pedagogía es baja y, por ende, su CPD en el dominio cognitivo también (BLÖMEKE et al., 2016).

Ahora, dado que las prácticas de instrucción se definen en parte por el conocimiento del dominio cognitivo, y a las capacidades de tomar decisiones, interpretar y percibir de los profesores (BLÖMEKE et al., 2016), es probable que estos estudiantes de pedagogía no tomen decisiones acertadas lo que se refleje en prácticas de instrucción que no tengan como propósito lograr aprendizajes y, por ende, el proceso de enseñanza no sea exitoso (STEIN; LANE, 1996).

Esto tiene directa relación con la eficacia del proceso de formación inicial de las tres universidades a las que pertenecen los estudiantes de pedagogía de la muestra. Aun cuando estas universidades y sus programas de formación tienen el más alto nivel de acreditación en Chile, esto, por lo visto en la descripción de las actividades, no asegura un aprendizaje de los elementos del dominio cognitivo de la CPD, uno de los propósitos de la FID. (BAUMERT; KUNTER, 2006; FERRINI-MUNDY et al., 2006; SHULMAN, 1986; BLÖMEKE et al., 2016; TATTO et al., 2012; WEINERT, 2001). De lo anterior, se hace necesario analizar las oportunidades que la FID otorga a los estudiantes de pedagogía en futuras investigaciones, conectando estas oportunidades con los desempeños de los estudiantes de pedagogía.

\section{Referencias}

ÁVALOS, B.; MATUS, C. La formación inicial docente en Chile desde una óptica internacional: Informe nacional del estudio internacional IEA TEDS-M. Santiago, n. 10, Chile: Ministerio de Educación, 2010.

BAUMERT, J.; KUNTER, M. Stichwort: Professionelle Kompetenz von Lehrkräften. Zeitschrift für Erziehungswissenschaft, Norway, v. 9, n. 4, p. 469-520, 2006.

BLÖMEKE, S. et al. The relation between content-specific and general teacher knowledge and skills. Teaching and Teacher Education, v. 56, p. 35-46, 2016.

BLÖMEKE, S. et al. Teacher change during induction: Development of beginning primary teachers' knowledge, beliefs and performance. International Journal of Science and Mathematics Education, Norway, v. 13, n. 2, p. 287-308, 2015.

BLÖMEKE, S. et al. TEDS-M 2008. Professionelle Kompetenz und Lerngelegenheiten angehender Primarstufenlehrkräfte im internationalen Vergleich. Múnster: Waxmann, 2010. 
BOSTON, M. et al. Comparison of mathematics classroom observation protocols. Mathematics Teacher Educator, Reston, v. 3, n. 2, p.154-175, 2015.

BOYD, D. et al. "Teacher Preparation and Student Achievment". Educational Evaluation and Policy Analysis, Washington, v. 31, n. 4, p. 416-440, 2009.

CLARKE, B. et al. Tasks in primary mathematics teacher education. 1. ed. New York: Springer, 2009.

CHAPMAN, O. Mathematics teacher educator's learning from research on their instructional practices. In: JAWORSKY, B.; WOOD, T. The Mathematics Teacher Educator as a Developing Professional. Handbook of Mathematics Teacher Education. Rotterdam, The Netherlands: Sense Publishers, 2008. p. 115-134.

DOYLE, W. Academic work. Review of educational research, Washington, v. 53, n. 2, p. 159-199, 1983.

DOYLE, W. Work in mathematics classes: The context of students' thinking during instruction. Educational Psychologist, Washington, v. 23, n. 2, p. 167-180, 1988.

FERRINI-MUNDY, J. et al. Knowing Mathematics: What We Can Learn from Teachers. PROM/SE Research Report. 2. ed. East Lansing: Michigan State University, 2006.

FINCH, W; FRENCH, B. Latent variable modeling with R. 1. ed. Routledge, 2015.

GAETE, A. et al. ¿Qué le piden los profesores a la formación inicial docente en Chile? Centro de Políticas Públicas UC, Santiago, n. 86. Temas de la Agenda Pública, 2016.

HAGGARTY, L.; PEPIN, B. An investigation of mathematics textbooks and their use in English, French and German classrooms: Who gets an opportunity to learn what? British educational research journal, vol. 28, no 4, p. 567-590, 2002.

HIEBERT, J.; LEFEVRE, P. Conceptual and Procedural Knowledge in Mathematics: An Introductory Analysis. In: HIEBERT, J. (Ed.). Conceptual and Procedural Knowledge: The Case of Mathematics. Hillsdale, NJ: Lawrence Erlbaum Associates, 1986. p. 1-27.

HIEBERT, J.; WEARNE, D. Instructional tasks, classroom discourse, and students' learning in second-grade arithmetic. American educational research journal, Washington, v. 30, n. 2, p. 393$425,1993$.

JAWORSKI, B. Theory and practice in mathematics teaching development: Critical inquiry as a mode of learning in teaching. Journal of mathematics teacher education, Reston, v. 9, n. 2, p. 187-211, 2007.

KÖNIG, J; BLÖMEKE, S. Future teachers' general pedagogical knowledge from a comparative perspective: does school experience matter? ZDM: The International Journal on Mathematics Education, Norway, v. 44, n. 3, p. 341-354, 2012.

KUNTER, M. et al. Professional competence of teachers: Effects on instructional quality and student development. Journal of Educational Psychology, Washington, v. 105, n. 3, p. 805, 2013.

LILJEDAHL, P. et al. Interweaving mathematics and pedagogy in task design: A tale of one task. Journal of Mathematics Teacher Education, Reston, v. 10, n. 4-6, p. 239-249, 2007. 
MA, L. Knowing and teaching elementary mathematics: Teachers' understanding of fundamental mathematics in China and the United States. 1. ed. Mahwah, NJ: Lawrence Erlbaum Associates, 1999.

MAGIDSON, J.; VERMUNT, J. K. Latent class models. In: Kaplan, D. (Ed.). The Sage handbook of quantitative methodology for the social sciences. Thousands Oakes: Sage, 2004. p. 175-198.

MAYRING, P. Qualitative content analysis - Theoretical foundation, basic procedures and software solution. Klagenfurt, 2014. Disponible en:

<https://www.psychopen.eu/fileadmin/user_upload/books/mayring/ssoar-2014-mayring-

Qualitative_content_analysis_theoretical_foundation.pdf>. Acceso en: 15 Marz. 2016.

MINEDUC. Informe de la Comisión sobre Formación Inicial Docente (documento no oficial).

Santiago de Chile: Ministerio de Educación, 2005.

MINEDUC. Resultados Evaluación Inicia 2014. Santiago: Ministerio de Educación, 2015.

NATIONAL COUNCIL OF TEACHERS OF MATHEMATICS (NCTM). Principles to actions:

Ensuring mathematical success for all. 1. ed. Reston, Va. 2014.

PEPIN, B. Enhancing mathematics/STEM education: a "resourceful" approach. 1.ed. Eindhoven: Technische Universiteit Eindhoven, 2015.

PEPIN, B. et al. Re-sourcing teacher work and interaction: new perspectives on resource design, use and teacher collaboration. ZDM: The International Journal on Mathematics Education, Norway, v. 45, n. 7, p. 929-943, 2013.

ROST, J. Test theory and test construction. 1. ed. Bern, Switzerland: Hans Huber, 2004.

SCHMIDT, W. H. et al. Many Visions, Many Aims: Volume 2: A Cross-National Investigation of Curricular Intensions in School Science. Springer Science \& Business Media, 1997.

SCHOENBERG, N. E.; RAVDAL, H. Using vignettes in awareness and attitudinal research. International Journal of Social Research Methodology, v. 3, n. 1, p. 63-74, 2000.

SHIMIZU, Y. et al. Mathematical tasks in classrooms around the world. 1. ed. Sense Publishers, 2010.

SHULMAN, L. Those who understand: Knowledge growth in teaching. Educational researcher, Washington, v. 15, n. 2, p. 4-14, 1986.

STEIN, M. K. et al. Building student capacity for mathematical thinking and reasoning: An analysis of mathematical tasks used in reform classrooms. American educational research journal, Washington, v. 33, n. 2, p. 455-488, 1996.

STEIN, M. K.; LANE, S. Instructional tasks and the development of student capacity to think and reason: An analysis of the relationship between teaching and learning in a reform mathematics project. Educational Research and Evaluation, Washington, v. 2, n. 1, p. 50-80, 1996.

STEIN, M. K.; SMITH, M. S. Mathematical tasks as a framework for reflection: From research to practice. Mathematics teaching in the middle school, Reston, v. 3, n. 4, p. 268-275, 1998.

SULLIVAN, P.et al. Teaching with tasks for effective mathematics learning. 1. ed. Berlin: Springer, 2012. 
TATTO, M. T. et al. Policy, Practice, and Readiness to Teach Primary and Secondary

Mathematics in 17 Countries: Findings from the IEA Teacher Education and Development Study in Mathematics (TEDS-MM). 1. ed. Amsterdam, The Netherlands: International Association for the Evaluation of Educational Achievement, 2012.

TATTO, M.T. et al. Teacher education and development study in mathematics (TEDS-M). Policy, practice, and readiness to teach primary and secondary mathematics. Conceptual framework. East Lansing, MI: Teacher Education and Development International Study Center, College of Education, Michigan State University, 2008.

THANHEISER, E. Developing prospective teachers' conceptions with well-designed tasks: Explaining successes and analyzing conceptual difficulties. Journal of Mathematics Teacher Education, Reston, v. 18, n. 2, p. 141-172, 2015.

WATSON, A.; OHTANI, M. Task design in mathematics education: An ICMI study. 1. ed. Berlin: Springer, 2015.

WEINERT, F. Concept of competence: A conceptual clarification. In: RYCHEN, D; SALGANIK, L. Defining and selecting key competencies. 1. ed. Göttingen: Hogrefe; Huber, 2001. p. 45- 66.

WILLIAMS, G.; CLARKE, D. The complexity of mathematics tasks. In: SCOTT, N;

HOLLINGSWORTH, H. Mathematics: Creating the future. Melbourne: Australia, 1997. p. 451-457.

ZASLAVSKY, O.; SULLIVAN, P. Constructing knowledge for teaching secondary mathematics: Tasks to enhance prospective and practicing teacher learning. 1. ed. Berlin: Springer, 2011.

Submetido em 16 de Julho de 2017. Aprovado em 15 de Fevereiro de 2018. 